\title{
Evaluation of the impact of Covid-19 infection on the evolution and prognosis of patients with acute leukaemia
}

\author{
Meilin Omer ${ }^{1}$, Ana M. Vladareanu ${ }^{2,3}$, Viola M. Popov ${ }^{1 *}$, Mihaela Andreescu ${ }^{1}$, \\ Lelia Iliescu ${ }^{4}$, Horia Bumbea ${ }^{2,3}$, Serban Dragosloveanu ${ }^{5}$ \\ 1. Hematology, Colentina Clinical Hospital, Romania \\ 2. Faculty of Medicine, Carol Davila University of Medicine and Pharmacy Bucharest Romania, \\ Romania \\ 3. Hematology, Emergency Clinical Hospital, Romania \\ 4. Intensive Care Unit 1, Colentina Clinical Hospital, Romania \\ 5. Orthopedy, Foisor Clinical Hospital, Romania
}

\begin{abstract}
Introduciton: COVID19 is one of the largest pandemics. Since December 2019 until now the coronavirus has infected over 131 million people. The mortality rate in the general population varies between 1 to 5\%, with a potential of over $30 \%$ in patients with neoplasms. Methods: The main objective of the study was to identify some peculiarities of the evolution, complications and treatment of patients with acute leukaemia and COVID-19. The study was retrospective and included 50 patients with acute leukaemia and COVID-19. Results: Recent administration of chemotherapy was identified in 20 patients with acute myeloblastic leukaemia and 4 patients with acute lymphoblastic leukaemia. The newly diagnosed patients or those undergoing intensive chemotherapy, in particular elderly patients, had a severe form of COVID-19 and an unfavourable evolution, and these clinical situations were identified as predictive factors for adverse outcomes. Patients with acute lymphoblastic leukaemia had a shorter survival curve compared to patients with acute myeloblastic leukaemia. Pneumonia was present especially in patients with acute myeloblastic leukaemia, most patients having over $30 \%$ of lung fields affected (55.26\%). Patients with an unfavourable outcome had significantly increased median values of C-reactive protein, procalcitonin and interleukin6. Conclusions: Patients with acute leukaemia, especially acute myeloblastic leukaemia who have been diagnosed with COVID-19 infection require special attention because they may associate complications and adverse outcomes of COVID-19. The results we obtained require evaluation in a larger group of patients and analysis in the follow-up period after COVID-19.
\end{abstract}

Keywords: acute leukemia, COVID-19, chemotherapy, aplasia, respiratory insuficiency

Received: 22 $2^{\text {nd }}$ July 2021; Accepted: $1^{\text {st }}$ October 2021; Published: $8^{\text {th }}$ October 2021

\footnotetext{
* Corresponding author: Viola M. Popov, Hematology, Colentina Clinical Hospital, Romania. E-mail: violamariap@gmail. com
} 


\section{Introduction}

COVID-19 is one of the largest pandemics. Since December 2019 until now the coronavirus has infected over 131 million people, summing over 2.8 million deaths. Between 25 to $50 \%$ of those exposed to COVID are asymptomatic; symptoms range from mild fever, dry cough, fatigue, headache, myalgia, and anosmia to severe pneumonia, disseminated intravascular coagulation associated with thrombotic events, cardiomyopathy, neurological disorders, multiple organ failure, and culminating with death. The mortality rate in the general population varies between 1 to $5 \%$, with a potential of over $30 \%$ in patients with neoplasms (1).

The likelihood of cancer patients to develop COVID-19 is high compared to the general population, in particular for those recently diagnosed, depending on risk factors such as age and other comorbidities, like uncontrolled type II diabetes and morbid obesity (in particular), chronic renal failure, BPOC, Down syndrome, pregnancy, severe cardiovascular damage, heart failure, coronary artery disease, solid organ post-transplant status, cardiomyopathy, and sickle cell disease. Moderate to severe asthma, cerebrovascular disease, cystic fibrosis, hematopoietic stem cell transplantation, HIV, use of corticosteroids, liver damage, hypertension, dementia, obesity (index $\geq 25$ but $<30 \mathrm{~kg} / \mathrm{m}^{2}$ ), pulmonary fibrosis, thalassemia, and type 1 diabetes could be other risk factors that contribute to severe evolution of COVID-19 (2). Studies have found that those with hematological diseases and lung cancer have an increased risk (3).

Thus, the possible risk factors in acute lymphoblastic leukemia are represented by: immunosuppression within the disease, along with chemotherapy, hypogammaglobulinemia, prolonged exposure to steroids, renal and pulmonary damage secondary to methotrexate therapy, heart failure secondary to anthracycline exposure.
There is also an increased risk for developing thrombosis and aspergillosis, and impairment of B cell function (1). For acute myeloblastic leukemia, possible risk factors are myelosuppression in the disease and post-chemotherapy status, heart failure secondary to anthracycline exposure, and lung damage secondary to midostaurin administration (1).

A New York hospital that treated cancer and COVID-19 patients found that the mortality rate was $37 \%$ (20/54 patients in total), showing that myeloid hematological disorders have a higher mortality rate tendency than lymphoid ones (4). Patients with severe leukopenia, especially severe lymphopenia $(<500 / \mathrm{uL})$, are prone to be transferred to an intensive care unit and have an unfavorable prognosis $(5,6)$.

Anticipated risk factors for COVID-19 infection in patients with acute leukemia include neutropenia, leukopenia, hypogammaglobulinemia, immunosuppression, tendency to hypercoagulability, organ dysfunction (cardiac, renal, hepatic, pulmonary), comorbidities, and age (7).

\section{Materials and methods}

The main objective of the study was to identify some peculiarities of the evolution, complications and treatment of patients with acute leukemia who associated COVID-19 infection. The study was retrospective and included 50 patients with acute leukemia and COVID-19, patients admitted to the Department of Hematology, Colentina Clinical Hospital between April 2020 and February 2021. The study was carried out after obtaining the approval of the Ethics Committee of the Colentina Hospital and having the patients' informed consent. The diagnosis of COVID-19 was established using PCR testing via nasal and pharyngeal swabs. The diagnosis of acute leukemia was established according to the WHO diagnostic criteria, the status of refractory/ progressive disease was considered when 
the disease evolved in spite of appropriate treatment schemes having been applied. The absence of any signs of disease was a criterion for classifying patients as being in remission. The study enrolled 38 patients with acute myeloblastic leukemia, 10 patients with acute lymphoblastic leukemia, and 2 patients with acute biphenotypic leukemia. Patients were diagnosed with acute leukemia prior to or concomitant with being admitted for COVID-19, some of them having multiple lines of treatment and being treated with life-saving chemotherapy schemes. The refractory form of acute myeloblastic leukemia was present in 12 patients, 1 patient had the refractory to acute lymphoblastic form of leukemia, and 1 patient had biphenotypic acute leukemia. Concomitant diagnosis of acute leukemia and COVID-19 was made in 6 patients with acute myeloblastic leukemia and 3 patients with acute lymphoblastic leukemia (Table 1). Data were recorded for the onset of hematological disease and COVID-19, treatment, medical history, and clinical course. Associated comorbidities were quantified using the Charlson Comorbidity Index.

We performed Chi-square tests to assess differences in demographic and clinical/ laboratory data between different groups. The study group was split using survival/ non-survival characteristics, AML/ ALL, and severity of COVID-19. Correlations between clinical parameters (medical history, symptoms at the onset, and laboratory values at the onset) and fatal outcome or the presence of complications/ severe disease stage in patients with COVID-19 were made using multivariate logistic analysis. Statistical analyses for establishing the difference between at least two of the assessed subgroups were performed using the Kruskal Wallis test. The rank test was used to correlate between two variables. The evaluation of the variables with an impact on survival was done by Cox proportional hazard. In all these tests we obtained a value that was considered statistically significant for $p$ $<0.5$. All statistical analyses were performed using MedCalc Statistical Software version 16.4.3 (MedCalc Software be, Ostend, Belgium; https:// www.medcalc.org; 2016)

\section{Results}

The mean age of the group of patients with acute myeloblastic leukemia was 64 years (54 to 67), and of those with acute lymphoblastic leukemia was 47 years (24 to 64). The characteristics of patient groups are presented in table 1 (Table 1). The management of these patients was difficult due to many factors: first of all, to the presence of a newly identified severe infection with insufficiently known treatment schemes, and also to the presence of an acute hemato-oncological disease for which treatment is urgent. Secondary to the administration of chemotherapy, patients presented severe pancytopenia that complicated the management of COVID-19 treatment. Recent administration of the chemotherapy scheme was identified in 20 patients with acute myeloblastic leukemia and 4 patients with acute lymphoblastic leukemia. Twelve patients with acute myeloblastic leukemia and 4 patients with acute lymphoblastic leukemia had severe forms of COVID-19 infection, these patients being admitted to the intensive care unit (32\%). The COVID-19 treatment applied to these patients was Remdesivir for patients in the first 8 days after the onset of symptoms or Dexamethasone and/ or Tocilizum$\mathrm{ab}$ for patients hospitalized for more than 8 days after the clinical onset of COVID-19.

Unfavorable evolution was identified in 10 patients with AML and 3 patients with ALL who needed hospitalization in the intensive care unit. In the group with moderate forms, only 2 patients with AML and one patient with ALL had unfavorable evolution.

It should be noted that 2 patients had 2 episodes of COVID infection, and for both of them the rein- 


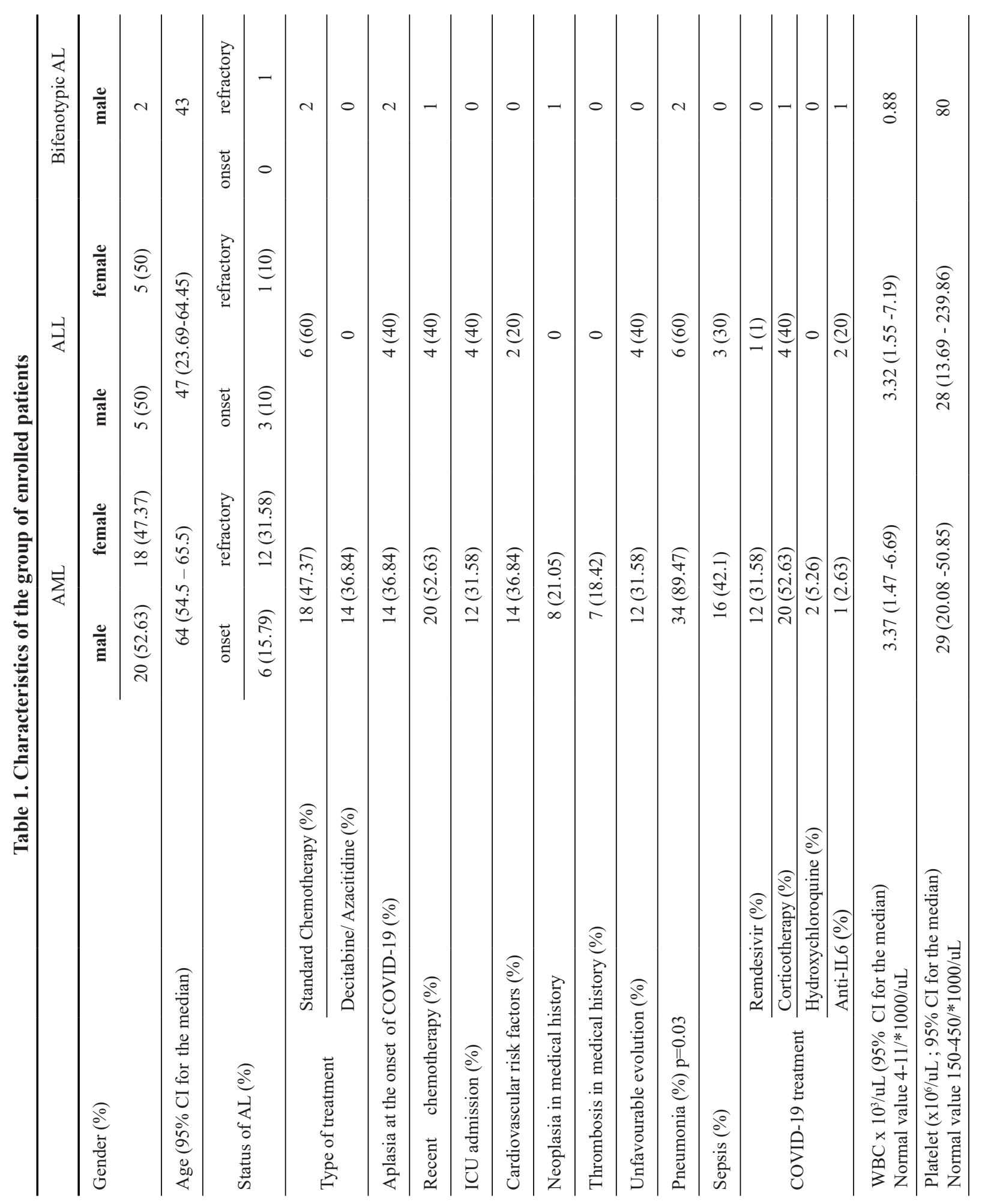




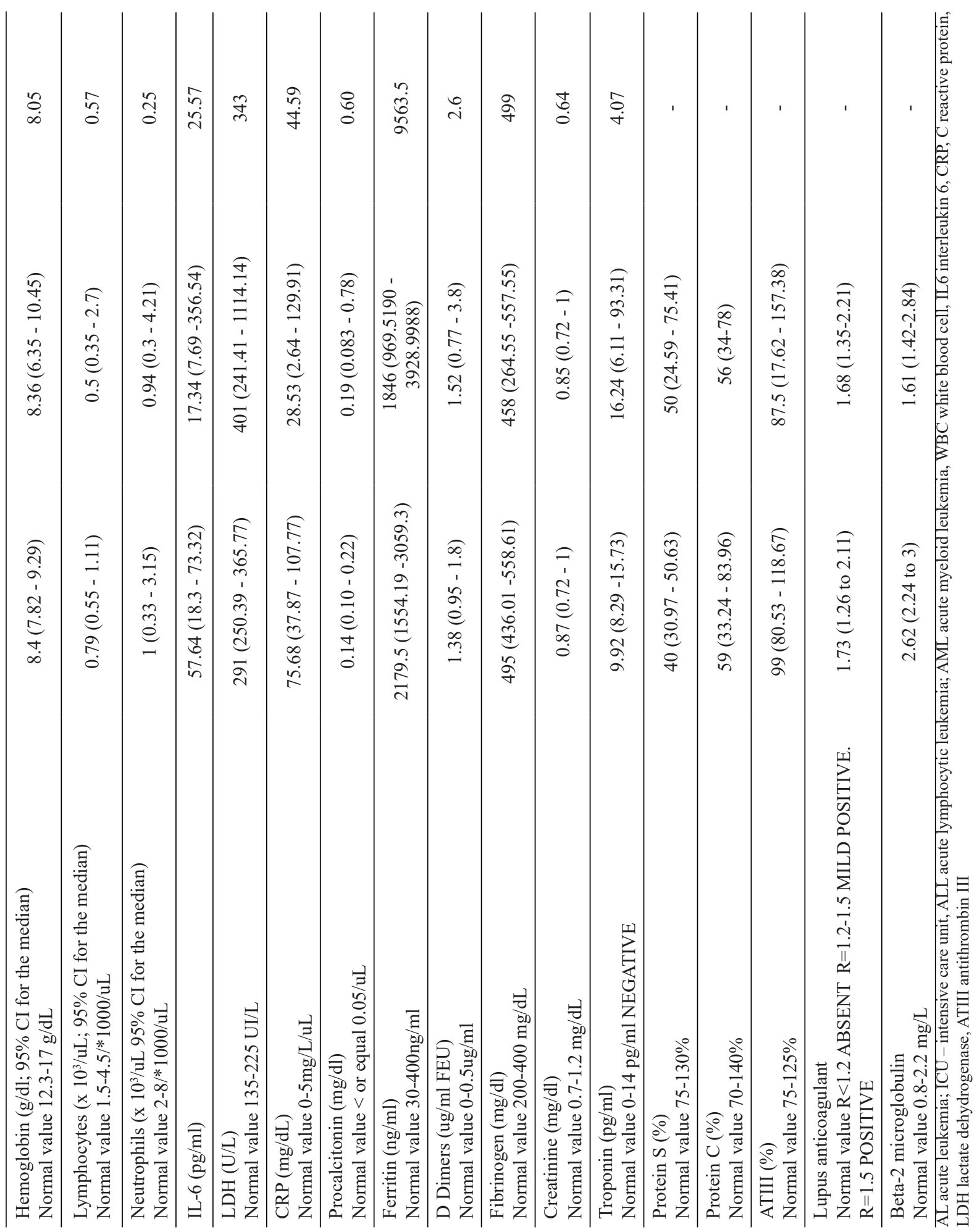


fection had an unfavorable evolution, the patients dying of septic complications. Another neoplasm associated with acute leukemia was present in 10 patients with acute myeloblastic leukemia. These were solid (colon, breast, prostate) or hematological tumors (MDS, MPNs). Vascular risk factors (hypertension, dyslipidemia, and hyperglycemia/ diabetes) were identified in 14 patients with acute myeloblastic leukemia and 2 patients with acute lymphoblastic leukemia. Thrombotic complications, strokes or myocardial infarction were present in 7 patients, all of which were identified in the patient's medical history. Hemorrhages due to severe thrombocytopenia and coagulation disorders were diagnosed in 7 patients with AML and 1 patient with ALL. The median value of the Charlson Comorbidity Index was 5 (min 2 max 9) for AML and $2(\min 2, \max 8)$ for ALL, $p=$ 0.03 . This index was identified as important to be calculated at the onset of COVID-19 in acute myeloid leukemia patients.

Patients with unfavorable outcomes had significantly increased median values of C-reactive protein, procalcitonin and interleukin 6: CRP $117.98 \mathrm{mg} / \mathrm{dl}(\min 2.22, \max 321.32)$ vs 34.45 $\mathrm{mg} / \mathrm{dl}(\min 1.55, \max 284.18) \mathrm{p}=0.03$ Fig.1; procalcitonin $0.29 \mathrm{mg} / \mathrm{dl}(\min 0.07, \max 12.6)$ vs $0.04 \mathrm{mg} / \mathrm{dl}(\min 1.55, \max 8.23) \mathrm{p}=0.01$; IL6

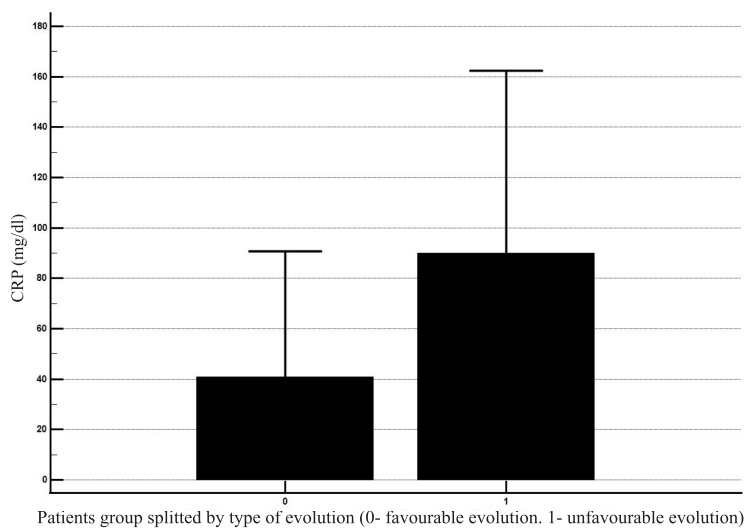

Fig. 1 The $\mathbf{C}$ reactive protein level compared to COVID-19 evolution ( 0 - favorable evolution; 1 deceased patients) $p=0.03$
$65.27 \mathrm{pg} / \mathrm{ml}(\min 8, \max 887.9)$ vs $18.46 \mathrm{mg}$ / dl (min 1.5, $\max 774.2) \mathrm{p}=0.05$ Fig. 2 The remaining hematological, biochemical, and coagulation parameters are not significantly different between groups identified by the type of evolution of COVID-19 (favorable/ unfavorable).

Severe forms of COVID-19 or the presence of newly diagnosed acute leukemia were predictive factors for adverse outcomes (hospitalization in ICU 12.03, p $=0.0005 \operatorname{Exp}(b)=20.9595 \% \mathrm{CI}$ of $\operatorname{Exp}(b)=3.75-116.9$, acute onset leukemia Wald 8.24, $\mathrm{p}=0.004, \operatorname{Exp}(\mathrm{b})=49.5,95 \% \mathrm{CI}$ of $\operatorname{Exp}(b)=3.45-709.77)$. Patients with acute lymphoblastic leukemia had a shorter survival curve compared to patients with acute myeloblastic leukemia, Chi-squared $=7.37, p=0.007$ Fig.3 .

Pneumonia was present especially in patients with acute myeloblastic leukemia, most patients having over $30 \%$ of lung fields affected $(55.26 \%)$. Patients with acute lymphoblastic leukemia and COVID-19 had pneumonia on imaging evaluation in fewer cases, $p=0.03$. Septic complications were present in both groups of patients and there were no statistically significant differences between the two groups of AML and ALL patients.

Without having statistical significance, due to the presence of a small number of patients, it can

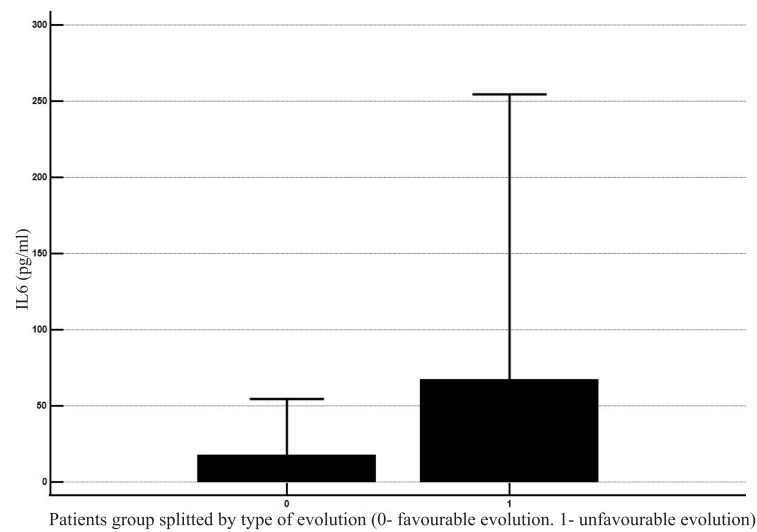

Fig.2 The interleukin-6 (IL6) level compared to COVID-19 evolution ( 0 - favorable evolution; 1 deceased patients) $p=0.05$ 


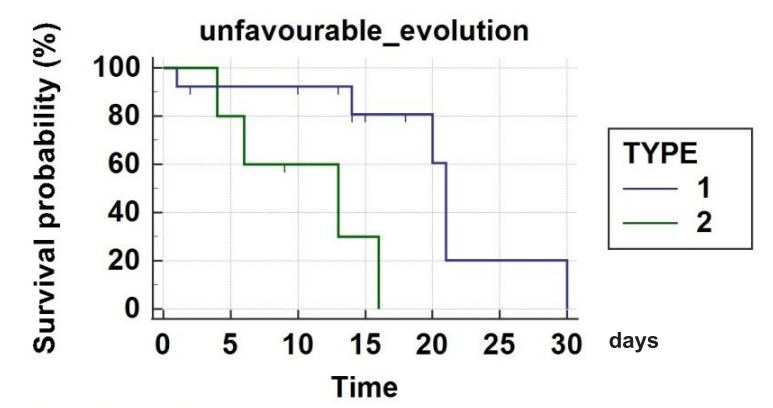

Number at risk

Group: 1

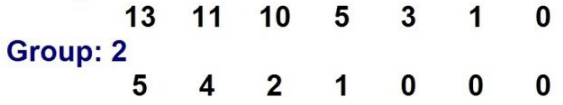

Fig.3 The survival analyses of acute leukemia patients during COVID-19 infection; survival time $=$ time between the onset of COVID-19 and death time (days), endpoint $=$ unfavorable evolution (1 acute myeloid leukemia, 2 acute lymphoid leukemia) Chi-squared $=7.37, p=0.007$

be seen that elderly patients who were recently diagnosed or those undergoing intensive chemotherapy followed by bone marrow aplasia, had a poor prognosis. These patients also needed intensive care services.

\section{Discussion}

Patients over 65 years of age and with hematological neoplasms have increased vulnerability to severe forms of COVID-19 (8). Patients with hematological neoplasms and COVID-19 infection have 4 times higher mortality rate compared to other COVID-19-positive patients. This increased mortality rate is especially present in patients who are refractory/ relapsed or who have undergone chemotherapy recently (9).

In our study, 8 patients had refractory forms of the disease, 3 of them with cytogenetic mutations, a criterion which included patients in the major risk class of evolution (Philadelphia chromosome or FLT3 mutation). There were also 2 patients who were included in the Venetoclax-Azacitidin protocol, the therapeutic life-saving scheme, secondary to the therapeutic failure of previous lines of treatment. This type of treatment was associated with favorable evolution of the COVID-19 infection.

It should be noted that all patients newly diagnosed with acute myeloblastic leukemia had unfavorable evolution, patients who either could not be prescribed medication due to the severe form COVID-19 or received standard $3+7$ chemotherapy followed by post-chemotherapy aplasia, all patients being over the age of 50 years. Data from the literature point to the unfavorable evolution of patients with newly diagnosed acute leukemia (10). In our study, we observed unfavorable evolution only for patients with AML. Patients with acute lymphoblastic leukemia and acute promyelocytic leukemia survived the COVID-19 episode.

Neutropenia and elevated values of CRP, procalcitonin, and D dimers are aggravating factors of evolution $(11,12)$. In the case of our group of patients we obtained significantly increased values of CRP in the group of patients with AL and unfavorable evolution compared to the CRP value in surviving patients with AL. Patients who did not survive the COVID-19 infectious episode had significantly higher levels of procalcitonin and interleukin 6 .

Neutropenia and leukopenia did not have a higher degree of severity in patients with acute leukemia with unfavorable evolution. However, patients with hematological neoplasms have a higher incidence of bacterial, fungal, and viral infectious complications compared to patients who do not have a history of malignant hematological diseases (12). The ratio of lymphocyte neutrophils, although identified by many studies as a predictive factor for severe evolution, in patients with acute leukemia did not show significant differences between the groups of analyzed patients (13-16).

Data from the literature confirm the favorable evolution of patients with AML receiving treat- 
ment with deacetylase inhibitors (17). Patients included in our study who received Azacitidin or Decitabin had favorable outcomes, except for one patient $(9.09 \%)$, who required hospitalization in the intensive care unit due to a severe form.

Having recently undergone chemotherapy has not been identified as an aggravating factor in the evolution of disease by some studies (18). During our study, the association with other factors (age, refractory status of the disease, and comorbidities) contributed to unfavorable evolution (17). Patients with AML and bone marrow allotransplant had unfavorable evolution possibly due to immunosuppressive treatment (19). Note the favorable evolution of the 2 patients with acute myeloblastic leukemia and bone marrow allotransplant performed before the COVID-19 infection. Less than 2 years later, the results were similar to those reported by other studies (17).

Patients with acute myeloblastic leukemia had significantly higher adverse outcomes compared to patients with acute lymphoblastic leukemia. In the group of patients with ALL, without statistical significance, the presence of the Philadelphia chromosome, life-saving chemotherapeutic treatments, and advanced age were indicators of unfavorable evolution. For the group of patients with acute promyelocytic leukemia, the refractory status of the disease was associated with unfavorable evolution.

\section{Conclusions}

Patients with acute leukemia, acute myeloblastic leukemia in particular, who have been diagnosed with COVID-19 infection, require special attention because they may associate complications and adverse outcomes of COVID-19. Intensive chemotherapy and aplasia have been aggravating factors, with the use of deacetylase inhibitors associated or not with Venetoclax giving a better prognosis in patients with AML and COVID-19.
It is crucial to calculate the Charlson Comorbidity Index at the onset of COVID-19. The results we obtained require evaluation in a larger group of patients and analysis in the follow-up period after COVID-19.

\section{Authors' contribution}

All authors designed the study, contributed to data collection, analysed, accessed and verified the data. All of them drafted the initial manuscript, contributed to the interpretation of the data, critically reviewed and approved the final draft of the manuscript and made the decision to submit the manuscript.

\section{Conflict of interest}

None to declare.

\section{References}

1. Paul S, Rausch CR, Jain N, Kadia T, Ravandi F, DiNardo C, et al. Treating Leukemia in the Time of COVID-19. Acta Haematol 2021;144:132-45. DOI: 10.1159/000508199

2. Centers for Disease Control and Prevention. Underlying medical conditions associated with high risk for severe COVID-19: Information for healthcare providers. Available at: https://www.cdc.gov/coronavirus/2019-ncov/hcp/clinical-care/underlyingconditions. html (Accessed on April 5, 2021).

3. Uzzo R, Kutikov A, Geynisman D, Michael B Atkins, Larissa Nekhlyudov, Richard A Larson et al. COVID-19: Risks for infection, clinical presentation, testing, and approach to infected patients with cancer, ed. UpToDate. Waltham, MA: UpToDate Inc. https:// www.uptodate.com (Accessed on 02 Apr, 2021).

4. Mehta V, Goel S, Kabarriti R, Cole D, Goldfinger M, Acuna-Villaorduna A, et al. Case Fatality Rate of Cancer Patients with COVID-19 in a New York Hospital System. Cancer Discov. 2020 Jul;10(7):935-41. DOI: 10.1158/2159-8290.CD-20-0516

5. Liu J, Li S, Liu J, Liang B, Wang X, Wang H, et al. Longitudinal characteristics of lymphocyte responses and cytokine profiles in the peripheral blood of 
SARS-CoV-2 infected patients. EBioMedicine. 2020 May;55:102763. DOI: 10.1016/j.ebiom.2020.102763

6. Ruan Q, Yang K, Wang W, Jiang L, Song J. Clinical predictors of mortality due to COVID-19 based on an analysis of data of 150 patients from Wuhan, China. Intensive Care Med. 2020 Apr 6;46(5):846-8. DOI: 10.1007/s00134-020-05991-x

7. Centers for Disease Control and Prevention. Science brief: Evidence used to update the list of underlying medical conditions that increase a person's risk of severe illness from COVID-19. Available at: https://www. cdc.gov/coronavirus/2019-ncov/hcp/clinical-care/underlying-evidence-table.html (Accessed on April 5, 2021).

8. El-Sharkawi D, Iyengar S. Haematological cancers and the risk of severe COVID-19: Exploration and critical evaluation of the evidence to date. Br J Haematol. 2020;190: 336-45. DOI: 10.1111/bjh.16956

9. Borah P, Mirgh S, Sharma SK, Bansal S, Dixit A, Dolai TK, et al, AIIMS Hematology Alumni Group. Effect of age, comorbidity and remission status on outcome of COVID-19 in patients with hematological malignancies. Blood Cells Mol Dis. 2021 Mar;87:102525. DOI: 10.1016/j.bcmd.2020.102525

10. Popov VM, Iliescu L, Balea MI, Horatiu Ioani, Mihaela Andreescu, Daniela Georgescu, et al. Predictive value of hematologic and coagulation parameters in assessment of severity and evolution of COVID-19 patients. Merit Research Journal of Medicine and Medical Sciences (ISSN: 2354-323X). 2020 October;8(10);605-15.

11. Pi-ana JL, Martino R, García-García I, Parody R, Morales $\mathrm{MD}$, Benzo $\mathrm{G}$. Infectious Complications Subcommittee of the Spanish Hematopoietic Stem Cell Transplantation and Cell Therapy Group (GETH). Risk factors and outcome of COVID-19 in patients with hematological malignancies. Exp Hematol Oncol. 2020 Aug 25;9:21. DOI: 10.1186/s40164-020-00177-z

12. Fattizzo B, Giannotta JA, Sciumè M, Cattaneo D, Bucelli C, Fracchiolla NS, et al. Reply to „COVID-19 in persons with haematological cancers": a focus on myeloid neoplasms and risk factors for mortality. Leukemia. 2020 Jul;34(7):1957-60. DOI: 10.1038/s41375020-0877-y

13. Liu J, Liu Y, Xiang P, Xiong H, Li C, Zhang M, et al. Neutrophil-to-lymphocyte ratio predicts critical illness patients with 2019 coronavirus disease in the early stage. J Transl Med. 2020;18(1):206. DOI: 10.1186/ s12967-020-02374-0

14. Rubinstein SM, Warner JL. COVID-19 and haematological malignancy: navigating a narrow strait. Lancet Haematol. 2020 Oct;7(10):e701-3. DOI: 10.1016/ S2352-3026(20)30252-0

15. Huang J, Lin H, Wu Y, Fang Y, Kumar R, Chen G, Lin S. COVID-19 in posttransplant patients-report of 2 cases. Am J Transplant. 2020 Jul;20(7):1879-81. DOI: 10.1111/ajt.15896

16. Shah GL, DeWolf S, Lee YJ, Tamari R, Dahi PB, Lavery JA, et al. Favorable outcomes of COVID-19 in recipients of hematopoietic cell transplantation. J Clin Invest. 2020 Dec 1;130(12):6656-67. DOI: 10.1172/ JCI141777

17. Ciccullo A, Borghetti A, Zileri Dal Verme L, Tosoni A, Lombardi F, Garcovich M, et al; GEMELLI AGAINST COVID Group. Neutrophil-to-lymphocyte ratio and clinical outcome in COVID-19: a report from the Italian front line. Int $\mathrm{J}$ Antimicrob Agents. 2020 Aug;56(2):106017. DOI: 10.1016/j.ijantimicag.2020.106017

18. Passamonti F, Cattaneo C, Arcaini L, Bruna R, Cavo $\mathrm{M}$, Merli $\mathrm{F}$, et al; ITA-HEMA-COV Investigators. Clinical characteristics and risk factors associated with COVID-19 severity in patients with haematological malignancies in Italy: a retrospective, multicentre, cohort study. Lancet Haematol. 2020 Oct;7(10):e737-45.

19. Chan AS, Rout A. Use of Neutrophil-to-Lymphocyte and Platelet-to-Lymphocyte Ratios in COVID-19. J Clin Med Res. 2020 Jul;12(7):448-53. DOI: 10.14740/ jocmr4240 